Article

\title{
Millimeter Wave Radiations Affect Membrane Hydration in Phosphatidylcholine Vesicles
}

\author{
Amerigo Beneduci $^{1}{ }^{1}$, Katia Cosentino ${ }^{1,2}$ and Giuseppe Chidichimo ${ }^{1}$
}

1 Department of Chemistry, University of Calabria, Via P. Bucci-Cubo 17/D, Arcavacata di Rende (CS) 87040, Italy; E-Mails: katia.cosentino@unical.it (K.C.); giuseppe.chidichimo@ unical.it (G.C.)

2 Adhesion and Inflammation, CNRS UMR 7333, INSERM U1067, Aix Marseille University, Luminy, Marseille 13009, France

* Author to whom correspondence should be addressed; E-Mail: amerigo.beneduci@ unical.it; Tel.: +39-0984-492-117; Fax: +39-0984-492-041.

Received: 8 May 2013; in revised form: 20 June 2013 / Accepted: 2 July 2013 /

Published: 9 July 2013

\begin{abstract}
A clear understanding of the response of biological systems to millimeter waves exposure is of increasing interest for the scientific community due to the recent convincing use of these radiations in the ultrafast wireless communications. Here we report a deuterium nuclear magnetic resonance spectroscopy $\left({ }^{2} \mathrm{H}-\mathrm{NMR}\right)$ investigation on the effects of millimeter waves in the $53-78 \mathrm{GHz}$ range on phosphocholine bio-mimetic membranes. Millimeter waves significantly affect the polar interface of the membrane causing a decrease of the heavy water quadrupole splitting. This effect is as important as inducing the transition from the fluid to the gel phase when the membrane exposure occurs in the neighborhood of the transition point. On the molecular level, the above effect can be well explained by membrane dehydration induced by the radiation.
\end{abstract}

Keywords: bio-mimetic membranes; phosphatidylcholine; millimeter wave exposure; deuterium quadrupole splitting; nuclear magnetic resonance

\section{Introduction}

The increasing interest in the biological effects of millimeter waves (MMWs; 30-300 GHz), expressed in recent years, is mainly due to the significant development of millimeter wave sources for ultrafast wireless communication systems [1]. Despite the low power density emitted from these 
sources (below $1 \mathrm{~mW} / \mathrm{cm}^{2}$ ), exposure to this radiation could be potentially dangerous for human beings [2-5]. Even though no significant thermal effects have been detected, several non-thermal biological effects have been claimed [2,3,5]. Reversible morphological and ultrastructural alterations were observed on different human tumor cell systems after long term exposure to radiation in the 53-78 GHz frequency range [6-8]. These effects were generally associated with a significant inhibition of cell proliferation [6-8]. In addition, it has been shown that these radiations might affect the kinetics of glucose metabolism in leukemia cells, enhancing the glycolytic aerobic pathway. This indicates that the exposed system needs to produce extra-bioenergy as a defense/reparatory response to the radiations, which results in a significant decrease in the proliferation rate without significant cell death [9]. Furthermore, it was also shown that these radiations can induce a reversible externalization of phosphatidylserine molecules in cells exposed in vitro, thereby affecting the structural state of phospholipids in biomembranes [10]. In contrast to these results, exposure performed at $42 \mathrm{GHz}$ and $60 \mathrm{GHz}$ did not result in any significant genotoxic effect or evidence of cellular stress [11-13]. For a detailed overview on the topic we refer readers to the recent review by Zhadobov et al. [5].

Strong evidence shows that biological membranes are likely to be the main target of millimeter waves with frequency in the range $1-80 \mathrm{GHz}$ [4]. In recent years, model membranes such as phospholipid monolayers [14], multilamellar [15] and unilamellar [16,17] phospholipid vesicles, have been increasingly used to investigate the effect of millimeter waves on the membrane structure and functions. Effects on the permeability of cationic liposomes were observed under exposure at $130 \mathrm{GHz}$ [16]. More recently, it has been shown that millimeter wave exposure in the frequency range 53-78 GHz affects the permeability properties of giant unilamellar vesicles (GUVs) to water under osmotic stress conditions and the kinetics of the aging processes in large unilamellar vesicles (LUVs) [17]. Both these effects have been explained in terms of a partial dehydration and consequent increased rigidity of the lipid membrane [17]. In addition, physical changes in GUVs, such as elongation, induced diffusion of fluorescent dye di-8-ANEPPS into the bilayer and increased attraction between vesicles, were observed under radiation exposure at $53.37 \mathrm{GHz}$ [18]. The action of the field on charged and dipolar residues, located at the membrane-water interface, is thought to be the major factor determining the overall perturbation of these vesicles [17,18]. This hypothesis is reinforced by ${ }^{2} \mathrm{H}-\mathrm{NMR}$ molecular spectroscopy analysis of the water bound to the membrane in multilamellar lipid vesicles exposed at 53-78 GHz, where an upward shift of the fluid-to-gel lipid transition temperature was observed [15].

Here we provide some results on the response of deuterium labeled 1,2-dimyristoyl-sn-glycero-3phosphatidylcholine $/{ }^{2} \mathrm{H}_{2} \mathrm{O}\left(\mathrm{DMPC} /{ }^{2} \mathrm{H}_{2} \mathrm{O}\right)$ membranes to millimeter waves $(53-78 \mathrm{GHz})$ at the physiologic temperature $\left(37{ }^{\circ} \mathrm{C}\right)$, obtained by ${ }^{2} \mathrm{H}-\mathrm{NMR}$ spectroscopy. Since the heavy water quadrupole splitting, $\Delta v_{\mathrm{q}}$, can be related to changes in the bilayer structures $[15,19,20]$, it is a useful probe of membrane surface geometry and membrane hydration. For a powder sample of multilamellar vesicles (MLVs), $\Delta v_{\mathrm{q}}$ is the distance (Hz) between the highest peaks of the powder spectrum (see Figure 1A) and is given by the sum of the contribution of water bound to different sites along the phosphocholine headgroup of the membrane [15,19,20]:

$$
\Delta v_{q}=\frac{3}{4} v_{q} \sum p_{b, i} S_{b, i}
$$


where, $v_{q}=220 \mathrm{KHz}$ is the water quadrupole coupling constant; $p_{b, i}$ and $S_{b, i}$ are, respectively, the fraction and the molecular order parameter of bound water at the $i$ th binding site. The last parameter takes the fast anisotropic molecular reorientations of water molecules at the membrane interface into account.

\section{Materials and Methods}

\subsection{Sample Preparation}

DMPC was purchased from Sigma Aldrich with a $98.8 \%$ purity and used without further purification. ${ }^{2} \mathrm{H}_{2} \mathrm{O}$ was obtained from Cambridge Isotope Inc. having a purity of $99.98 \%$. DMPC phospholipids labeled with deuterium on the $\mathrm{C} 2$ carbon atoms of the sn1 and sn2 chains (DMPC-d4) were synthesized as described elsewhere [21] and purified by column chromatography. Their purity was further checked by thin layer chromatography and used without further purification. Mixtures of pure DMPC with deuterium labeled DMPC were prepared by solubilization in chloroform. The solvent was removed by blowing a gentle stream of nitrogen over the sample and then placing it under high vacuum overnight. A white dry fluffy powder was thus obtained. DMPC powders were dried to a constant weight in vacuum at room temperature. Multilamellar vesicles (MLVs) were prepared by adding an aliquot of water (either $\mathrm{H}_{2} \mathrm{O}$ or $\mathrm{D}_{2} \mathrm{O}$ ) to a known amount of dry phospholipids in a $5 \mathrm{~mm}$ quartz tube that was hermetically sealed. Samples were weighted and stored at $37{ }^{\circ} \mathrm{C}$ for three days. Before performing the exposure treatment, ${ }^{2} \mathrm{H}-\mathrm{NMR}$ spectra were recorded over $48 \mathrm{~h}$ to ensure the time stability of the observed splitting. After any exposure, samples were weighted to check out for water evaporation.

\section{2. ${ }^{2}$ H-NMR Acquisitions}

DMPC/ $\mathrm{D}_{2} \mathrm{O}$ and DMPC-d4/ $\mathrm{H}_{2} \mathrm{O}$ phase transition were monitored with a precision of $0.2{ }^{\circ} \mathrm{C}$, by a Bruker VT 2000 unit (Bruker Biospin, Germany). Samples were brought into the fluid phase, above $\mathrm{T}_{\mathrm{m}}$, and their temperature was gradually decreased in steps of $0.2^{\circ} \mathrm{C}$ in order to enter the gel phase. For each new $\mathrm{T}$ value, the system was equilibrated for at least $30 \mathrm{~min}$ prior to acquisition. The reverse process (gel-to-fluid phase) was studied by increasing the temperature in the same way. For DMPC-d4 samples, spectra were acquired using a spectral width of $100 \mathrm{KHz}$ and accumulating 3000-7000 FIDs. Spectra were de-Paked using the dmfit free program [22] according to the procedures described by the authors. FIDs were apodized with an exponential decay corresponding to a line broadening of $200 \mathrm{~Hz}$, prior to perform the FT.

Segmental order parameters of the acyl chains of the phospholipids were calculated by the quadrupole splitting of the deuterium nuclei in the $\mathrm{C}-{ }^{2} \mathrm{H}$ bond, expressed as $\left|\Delta v_{Q}\right|=\frac{3}{2} v_{Q}\left|S_{2}^{C D}\right|\left|\frac{3 \cos ^{2} \vartheta-1}{2}\right|$, where $v_{Q}$ is the quadrupole coupling constant equal to $167 \mathrm{KHz}$ and $\vartheta$ the angle between the director of the lamellar phase and the static magnetic field. $\Delta v_{Q}$ was measured as the distance of the two inner singularities of the powder spectrum $\left(\vartheta=90^{\circ}\right)$ [23-26]. 


\subsection{Real-Time NMR Acquisition under Exposure}

MMW exposure was performed by means of a microwave wide-band generator (Amphit-32, MicroMedTech, Nizhny Novgorod, Russia) in the 53.57-78.33 GHz frequency (f) range and incident power densities (IPD) of $0.0035-0.010 \mathrm{~mW} / \mathrm{cm}^{2}$. Further experiments were carried out irradiating the samples with monochromatic millimeter sources at $53.37 \mathrm{GHz}, 62.10 \mathrm{GHz}$ and $65.00 \mathrm{GHz}$ (IMG series, MicroMedTech, Nizhny Novgorod, Russia).

To allow real time detection of NMR spectra during exposure, the MMW generator was connected to a diamagnetic cylindrical waveguide of variable length terminating with a dielectric Teflon antenna inserted into the NMR probe with a vertical coil (Figure 1). Samples were subjected either to MMW radiation or to sham exposure, which corresponds to the same set-up but with the generator turned off (Incident power density $=0$ ). NMR spectra were acquired in real-time in strictly controlled temperature conditions.

Figure 1. Exposure set-up showing the millimeter-waves applicator connected to a diamagnetic cylindrical waveguide inserted into to the nuclear magnetic resonance (NMR) magnet (A); The waveguide terminates with a dielectric Teflon antenna located in the NMR probe over the sample $(\mathbf{B})$. Model of the scenario for the dosimetric assessment: the millimeter waves (MMWs) applicator inserted into a cuvette with the exposed sample.

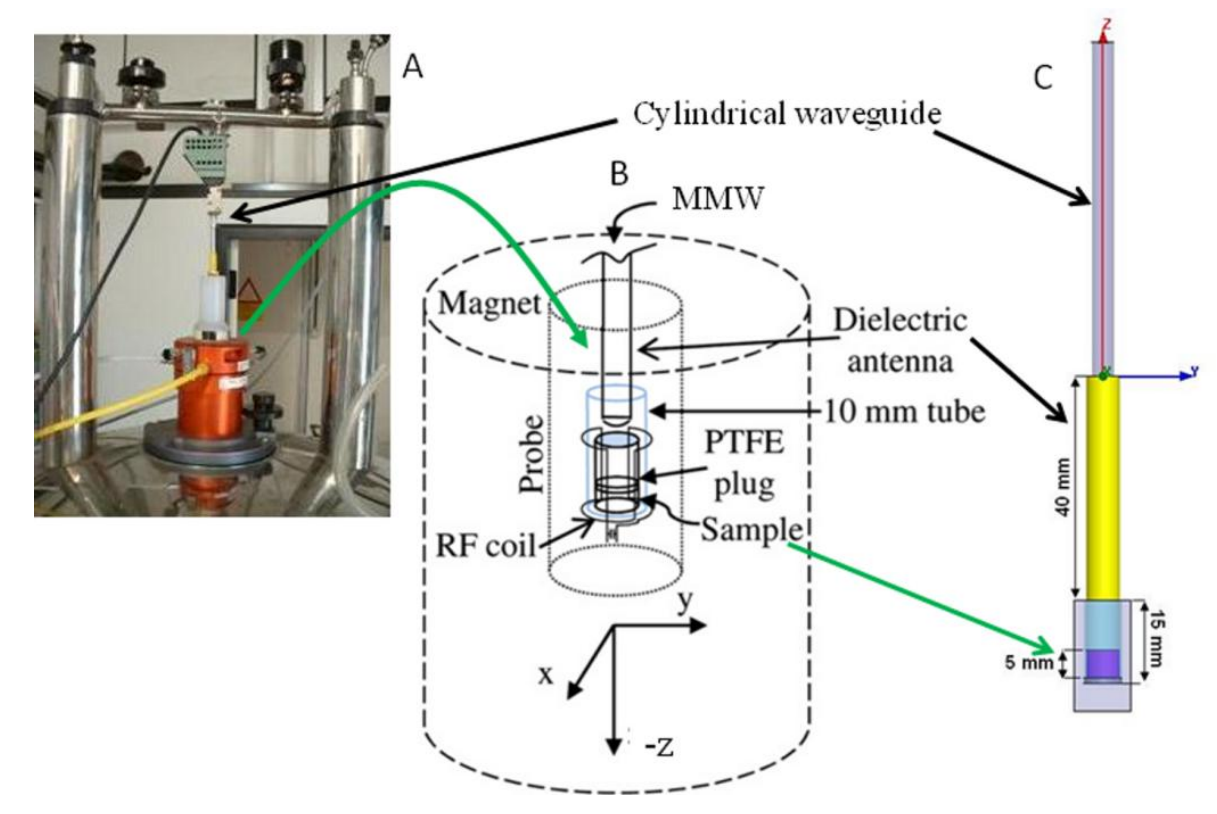

\section{Results and Discussion}

\subsection{Exposure at 53-78 GHz Causes a Time-Dependent Decrease of the Quadrupole Splitting}

${ }^{2} \mathrm{H}-\mathrm{NMR}$ spectra of the DMPC/ ${ }^{2} \mathrm{H}_{2} \mathrm{O}$ membrane acquired at $37{ }^{\circ} \mathrm{C}$ before and after $4 \mathrm{~h}$ of millimeter wave wide-band exposure in the range $53-78 \mathrm{GHz}$ are compared in Figure 2A. The radiation induced a decrease of the water quadrupole splitting of about $25 \%$ (Table 1). This effect could be observed only after some exposure time (up to several hours). 
Spectra acquired at $27^{\circ} \mathrm{C}$ under the same exposure conditions, showed a similar trend (Figure 2B) [15]. Notably, in this case, the effect of the quadrupole splitting decrease is large enough to induce the fluid-to-gel phase transition at $27{ }^{\circ} \mathrm{C}$ (Figure 2C), where in the sham exposed (control) system the main phase transition occurs at $25.5^{\circ} \mathrm{C}$.

It is worth noting that the decrease of the water quadrupole splitting is reversible and the membrane system relaxes back to its pre-exposure state after a few hours from the end of the exposure. The time-dependent nature of this phenomenon suggests the hypothesis that the energy provided by the radiation is accumulated by the system over time until it becomes sufficient to induce the observed effects.

This idea, first conjectured by Fröhlich [27], has recently been revised by Reimers et al. [28], but still needs to be definitely proved.

Figure 2. Effects of millimeter waves $(53-78 \mathrm{GHz})$ on deuterium labeled 1,2-dimyristoyl-sn-glycero-3-phosphatidylcholine (DMPC) vesicles measured as real-time changes of the ${ }^{2} \mathrm{H}-\mathrm{NMR}$ line shapes at $37^{\circ} \mathrm{C}(\mathbf{A})$ and $27^{\circ} \mathrm{C}(\mathbf{B})$ during exposure. The heavy water quadrupole splitting $\left(\Delta v_{\mathrm{q}}\right)$ is defined as the distance (in Hz) between the peaks of the spectrum. Quadrupole splitting behavior of DMPC/ ${ }^{2} \mathrm{H}_{2} \mathrm{O}$ multilamellar vesicles (MLVs) $\left(n_{\mathrm{w}}=11\right)$ under sham $(\bullet)$ and MMWs exposure $(\boldsymbol{\bullet})$ in the transition region $\left(\mathrm{T}_{\mathrm{m}}=\right.$ phase transition temperature).
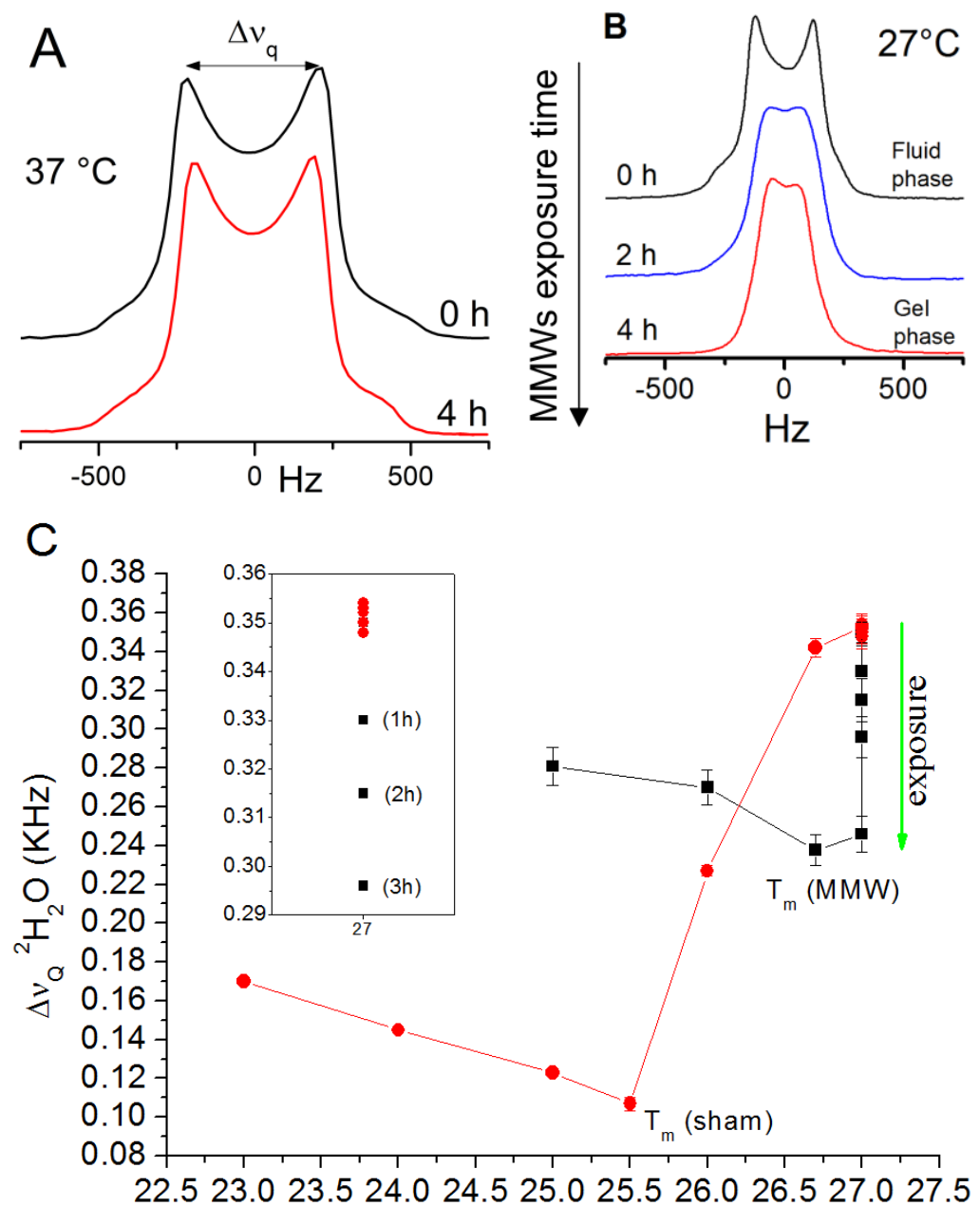

Temperature $\left({ }^{\circ} \mathrm{C}\right)$ 


\subsection{The Observed Effects Have a Non-Thermal Nature}

To prove the nonthermal nature of the effects observed under exposure, we have followed the change in the quadrupolar splitting by increasing (and decreasing) the temperature in the range 30-50 ${ }^{\circ} \mathrm{C}$ (Figure 3A). A temperature increase induces an increase in the quadrupolar splitting (Figure 3B). Therefore the decrease in the quadrupolar splitting observed under exposure (Figure 2) cannot be due to heating effects. Further, accurate dosimetric analysis (see supplementary information) confirms that only negligible thermal effects were induced on the membrane during irradiation. Indeed, in the exposure conditions described, an average whole sample average specific absorption rate (SAR) ranging from 1 to $3 \mathrm{~mW} / \mathrm{kg}$ was calculated, depending on the frequency and on the sample thickness, with average time rate of initial temperature rise of the order of $10^{-6}-10^{-7} \mathrm{~K} / \mathrm{s}$. Furthermore, the largest estimated thermal gradient $\left(74 \mathrm{GHz}, 20 \mu \mathrm{W}\right.$ output power) was $\sim 0.08<0.1{ }^{\circ} \mathrm{C}$ (see supplementary materials and [15]).

Figure 3. Temperature effect on the heavy water quadrupole splitting $\left(\Delta v_{\mathrm{q}}\right)$ of DMPC vesicles with a water/lipid mole ratio $n=12$ in the range $30-50{ }^{\circ} \mathrm{C}$. (A) Heating ( $($ ) and cooling ( $\square$ ) curves and (B) corresponding ${ }^{2} \mathrm{H}-\mathrm{NMR}$ lineshape changes for selected temperatures.
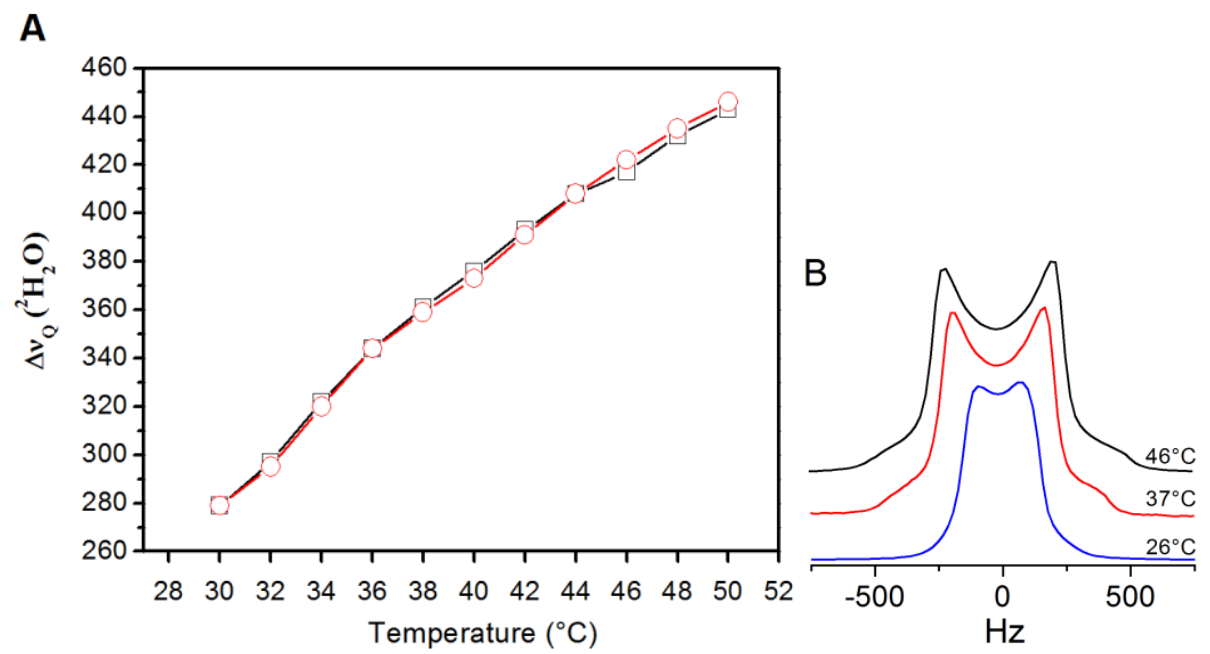

\subsection{Dependence on the Water/Lipid Mole Ratio}

Figure 4 shows the heavy water quadrupole splitting reduction $(\%)$ induced by $4 \mathrm{~h}$ millimeter wave exposure as a function of the membrane hydration regime, i.e., the water/lipid mole ratio $\left(n_{\mathrm{w}}\right)$. It can be seen that the effect of the radiation increases with the increase in $n_{\mathrm{w}}$, suggesting that this effect is related to some change in the membrane hydration. 
Figure 4. Heavy water quadrupole splitting reduction induced by $4 \mathrm{~h}$ MMWs exposure in the wide-band mode on DMPC/ ${ }^{2} \mathrm{H}_{2} \mathrm{O}$ MLVs samples with different water/mole lipid ratio $\left(n_{\mathrm{w}}\right)$. Points and error bars are, respectively, means and standard deviations relative to three independent experiments.

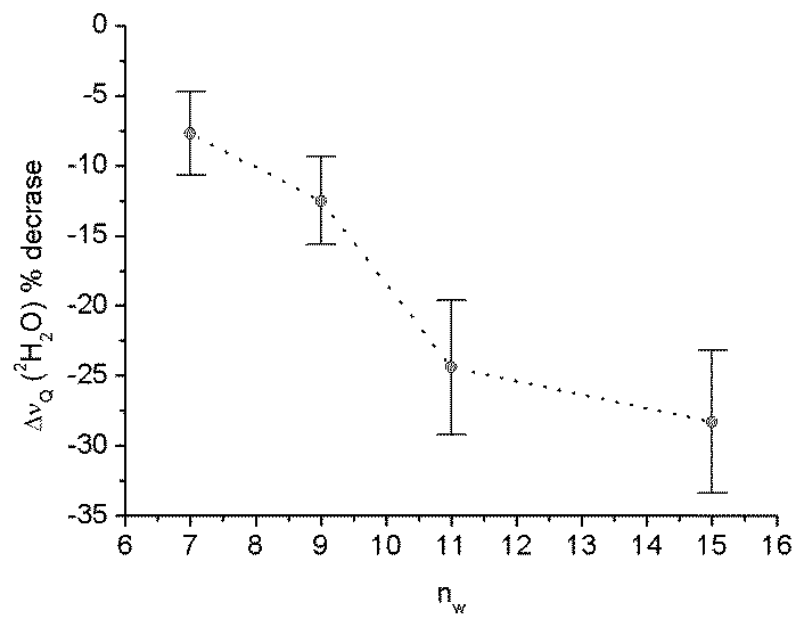

\subsection{Frequency-Dependent Effects}

In order to probe whether the above effects were frequency-specific in the range of frequencies used, we exposed the sample to three different frequency points: $53.37 \mathrm{GHz}, 62.1 \mathrm{GHz}$ and $65.00 \mathrm{GHz}$.

Table 1. Effects induced by extremely high frequency millimeter waves on DMPC multilamellar vesicles $\left(n_{\mathrm{w}}=11\right)$ in the fluid phase at $37{ }^{\circ} \mathrm{C}$ probed by ${ }^{2} \mathrm{H}-\mathrm{NMR}$ spectroscopy.

\begin{tabular}{cccc}
\hline \multirow{2}{*}{ Exposure frequency mode } & \multicolumn{2}{c}{ Exposure conditions } & Effects \\
\cline { 2 - 4 } & Frequency $(\mathrm{GHz})$ & $(\mathrm{SAR})^{\mathrm{a}} \mathrm{mW} / \mathrm{kg}$ & $\Delta \mathrm{v}_{\mathrm{q}}$ Decrease $(\%)^{\mathrm{b}}$ \\
\hline \multirow{2}{*}{ wide-band } & $53-78$ & $1-3$ & $25 \pm 5 \%$ \\
\multirow{2}{*}{ frequency point } & 53.37 & 2.7 & $\mathrm{nd}^{\mathrm{c}}$ \\
& 62.10 & 1.2 & $22 \pm 5$ \\
& 65.00 & 1.0 & $\mathrm{nd}^{\mathrm{c}}$ \\
\hline
\end{tabular}

${ }^{\mathrm{a}}$ As calculated in [12]; ${ }^{\mathrm{b}}$ Values averaged over three independent experiments; ${ }^{\mathrm{c}}$ Not detected.

Table 1 summarizes the changes detected in the heavy water NMR powder spectra on DMPC multilamellar vesicles by the different exposure conditions tested, after $4 \mathrm{~h}$ of continuous wave exposure. Among the three single-mode frequencies, only the exposure at $62 \mathrm{GHz}$ induced a change of the quadrupole splitting similar to the one observed under wide-band mode exposure conditions. This preliminary result suggests a specific frequency response of the investigated membrane model system in the 53-78 GHz range. 


\subsection{Millimeter Waves Change the Bound Water Partitioning at the Membrane Polar Interface} Increasing the Membrane Rigidity

The observed water quadrupole splitting decrease can be interpreted as a change of the water distribution among the various binding sites of the phosphocholine headgroup, i.e., as a change of $p_{b, i}$ (Equation (1)).

The preliminary data reported in Table 2 on DMPC membrane labeled with deuterium in the acyl chains, show that this water distribution rearrangement at the headgroups is coupled to a significant change of the $\mathrm{C} 1-\mathrm{C} 2$ segment orientation of the sn2 chain. Table 2 reports the $\mathrm{S}_{2}{ }^{\mathrm{CD}}$ segmental order parameters for the DMPC-d4/ $\mathrm{H}_{2} \mathrm{O}$ bio-membrane. Under exposure, we have observed a significant increase of the absolute value of the intramolecular $\mathrm{CD}$ bond order parameter relative to the deuteron pro- $\mathrm{R}$ in position $\mathrm{C} 2$ of the sn2 phospholipid chain (Table 2). It is worth noting that the change induced on this parameter increases with increasing $n_{\mathrm{w}}$ (Table 2), thus supporting the results of Figure 4. The sn2 pro-R parameter is very sensitive to structural changes of the headgroups conformation (especially near the phase transition) and its increase suggests that the beginning of the sn2 chain tends to be more extended into the bilayer core, reflecting an increase in the chain packing [29].

On the other hand, this interpretation is consistent with the increase of the phospholipid lateral pressure in phosphocholine monolayers exposed at $61 \mathrm{GHz}$ and at a few $\mathrm{pW} / \mathrm{cm}^{2}$ [14] as well as to a decrease of the membrane permeability to water as observed in GUVs under osmotic stress [17].

Table 2. $\mathrm{S}_{2}{ }^{\mathrm{CD}}$ order parameters for the position $\mathrm{C} 2$ of the sn1 and sn2 lipid chains for two different hydration regime $\left(n_{\mathrm{w}}\right)$

\begin{tabular}{|c|c|c|c|c|}
\hline \multirow{4}{*}{$\begin{array}{c}\text { Order parameter } \\
\mathrm{S}_{2}{ }^{\mathrm{CD}} \\
\text { chain segment } \\
\text { sn1 }\end{array}$} & \multicolumn{4}{|c|}{$\mathrm{DMPC} / \mathrm{H}_{2} \mathrm{O}$} \\
\hline & \multicolumn{2}{|c|}{$n_{\mathrm{w}}=11$} & \multicolumn{2}{|c|}{$n_{\mathrm{w}}=17$} \\
\hline & Sham & Exposed & Sham & Exposed \\
\hline & -0.210 & -0.211 & -0.198 & -0.197 \\
\hline sn2 (pro-R) & $-0.156(0.001)$ & $-0.162(0.003)$ & $-0.130(0.002)$ & $-0.136(0.002)$ \\
\hline sn2 (pro-S) & 0.112 & 0.110 & 0.090 & 0.090 \\
\hline
\end{tabular}

Note: $\mathrm{sn} 2$ (pro-R) values are expressed as mean (sd) of three independent experiments.

According to the literature, the two deuterons on position $\mathrm{C} 2$ of the sn 1 chain are magnetically equivalents and they exhibit unique order parameter with negative sign due to geometrical reasons [29]. In fact, in the all-trans chain conformation, typical of the fluid phase, the C-D bonds form approximately tetrahedral angle with respect to the normal to the bilayer, having the beginning of the sn 1 chain an extended average conformation. Conformational disorder is induced by increasing the temperature, as it is shown by the markedly decrease of the $\mathrm{sn} 1-\mathrm{S}_{2}{ }^{\mathrm{CD}}$, showing that the $\mathrm{sn} 1-\mathrm{S}_{2}{ }^{\mathrm{CD}}$ is very sensitive to thermal variations [29]. In the sn2 chain, the two deuterons on the alpha carbon atom are magnetically nonequivalent and two different order parameters are observed: one positive, assigned to the pro-S deuteron and the other one negative, associated to the pro- $\mathrm{R}$ deuteron. The non-equivalence of the deuterons has been used to determine the average orientation of the $0=\mathrm{C}-\mathrm{C}$ group linked to the glycerol backbone [29]. It has been shown that, due to allowed trans-gauche isomerization of the $\mathrm{C} 1-\mathrm{C} 2$ bond, this segment of the sn2 chain adopts a favorable, thermally insensitive bent conformation 
with respect to the normal to the bilayer [29]. In particular, the pro-S $\mathrm{S}_{2}{ }^{\mathrm{CD}}$ is not sensitive to either thermal variations or structural changes [29]. On the other hand, the pro- $R \mathrm{~S}_{2}{ }^{\mathrm{CD}}$ is poorly affected by thermal variation (up to $40{ }^{\circ} \mathrm{C}$ ) while it is very sensitive to structural changes that affect the geometrical orientation of the $\mathrm{C} 1-\mathrm{C} 2$ segment, which is the case when the system approaches the phase transition [29]. This parameter can be therefore used as a peculiar sensor for the reorientation of the beginning of the sn 2 chain. From Table 2 it can be seen that radiation exposure significantly increases the absolute value of the pro- $\mathrm{R}_{2}{ }^{\mathrm{CD}}$ order parameter, after about $4 \mathrm{~h}$ of exposure. Interestingly, it does not affect either the pro-S $\mathrm{S}_{2}{ }^{\mathrm{CD}}$ or the $\mathrm{sn} 1-\mathrm{S}_{2}{ }^{\mathrm{CD}}$ parameters. In our exposure experiment, no variation of temperature and composition of the sample were allowed, thus the pro-S $\mathrm{S}_{2}{ }^{\mathrm{CD}}$ and the $\mathrm{sn} 1-\mathrm{S}_{2}{ }^{\mathrm{CD}}$ do not change significantly. On the other hand, a larger value of the $\mid$ pro $-\mathrm{RS}_{2}^{\mathrm{CD}} \mid$ means that the $\mathrm{C}-\mathrm{D}$ bond undergoes restricted dynamics of the trans-gauche conformational isomerization around the $\mathrm{C} 1-\mathrm{C} 2$ bond. This is related to a change in the orientation of the C1-C2 segment of the sn2 chain that adopts a less bent orientation [29]. Therefore, in the exposed bio-membranes, the sn 2 chain tends to be more extended along the normal to the bilayer. We then surmise that the structural changes of the headgroups conformation related to the radiation induced changes in the water partitioning at the membrane interface, are coupled to an increase of the rigidity of the bilayer.

\section{Conclusions}

The above results can be summarized as follows:

(1) The time-dependent nature of the effect suggests that accumulation phenomena occur, i.e., that the electromagnetic energy is mainly stored in the system during the exposure as chemical potential and is not simply thermalized.

(2) The effect is amplified in proximity to the membrane phase transition point. It suggests that cooperative phenomena occur, due to long range interaction among the membrane subunits.

(3) The MMWs-induced water quadrupole splitting reduction suggests a change in the water partitioning at the membrane interface.

(4) The strong dependence of the exposure effects on the water/lipid mole ratio reveals the critical role of water in mediating the MMWs/biomembrane interaction: as the water/lipid mole ratio increases, increasing mm-wave energy is deposited into the sample, since water strongly absorbs the radiation, leading to a greater effect.

Altogether, these effects indicate that extensive exposure to millimeter waves $(53-78 \mathrm{GHz})$ causes molecular changes at the water-bilayer interface. These changes can have far reaching consequences on the properties and function of biological membranes. Lateral diffusion of lipids and proteins inside the membrane, passive diffusion of small molecules through the bilayer, conformational changes of proteins inside the membrane, are all processes which are strictly dependent on the membrane hydration and permeability, and any change in the membrane properties can affect regulations and functions of biological cells. 


\section{Acknowledgements}

The authors would like to thank P.W. Westermann (North Eastern Ohio Universities College of Medicine) for providing the phospholipid DMPC-d4.

\section{Conflict of Interest}

The authors declare no conflict of interest.

\section{References}

1. Kleine-Ostmann, T.; Nagatsuma, T. A Review on Terahertz Communications Research. J. Infrared Millim. Terahertz Waves 2011, 32, 143-171.

2. Pakhomov, A.G.; Akyel, Y.; Pakhomova, O.N.; Stuck, B.E.; Murphy, M.R. Current state and implications of research on biological effects of millimeter waves: A review of the literature. Bioelectromagnetics 1998, 19, 393-413.

3. Belyaev, I.Y. Non-thermal Biological Effects of Microwaves. Microw. Rev. 2005, 11, 13-29.

4. Ramundo-Orlando, A. Effects of Millimeter Waves Radiation on Cell Membrane-A Brief Review. J. Infrared Millim. Terahertz Waves 2010, 31, 1400-1411.

5. Zhadobov, M.; Chahat, N.; Sauleau, R.; le Quement, C.; le Drean, Y. Millimeter-wave interactions with the human body: State of knowledge and recent advances. Int. J. Microw. Wirel. Technol. 2011, 3, 237-247.

6. Beneduci, A.; Chidichimo, G.; Tripepi, S.; Perrotta, E.; Cufone, F. Antiproliferative effect of millimeter radiation on human erythromyeloid leukemia cell line K562 in culture: Ultrastructural- and metabolic-induced changes. Bioelectrochemistry 2007, 70, 214-220.

7. Beneduci, A.; Chidichimo, G.; de rose, R.; Filippelli, L.; Straface, S.V.; Venuta, S. Frequency and Irradiation Time-dependant Antiproliferative Effect of Low-power Millimeter Waves on RPMI 7932 Human Melanoma Cell Line. Anticancer Res. 2005, 25, 1023-1028.

8. Beneduci, A.; Chidichimo, G.; Tripepi, S.; Perrotta, E. Transmission Electron Microscopy Study of the Effects Produced by Wide-band Low-power Millimeter Waves on MCF-7 Human Breast Cancer Cells in Culture. Anticancer Res. 2005, 25, 1009-1013.

9. Chidichimo, G.; Beneduci, A.; Nicoletta, M.; Critelli, M.; de Rose, R.; Tkatchenko, Y.; Abonante, S.; Tripepi, S.; Perrotta, E. Selective Inhibition of Tumoral Cells Growth by Low Power Millimeter Waves. Anticancer Res. 2002, 22, 1681-1688.

10. Szabo, I.; Kappelmayer, J.; Alekseev, S.I.; Ziskin, M.C. Millimeter wave induced reversible externalization of phosphatidylserine molecules in cells exposed in vitro. Bioelectromagnetics 2006, 27, 233-244.

11. Logani, M.K.; Bhanushali, A.; Ziskin, M.C.; Prihoda, T.J. Micronuclei in Peripheral Blood and Bone Marrow Cells of Mice Exposed to $42 \mathrm{GHz}$ Electromagnetic Millimeter Waves. Radiat. Res. 2004, 161, 341-345.

12. Zhadobov, M.; Nicolaz, C.N.; Sauleau, R.; Desmots, F.; Thouroude, D.; Michel, D.; le Drean, Y. Evaluation of the Potential Biological Effects of the 60-GHz Millimeter Waves Upon Human Cells. Antennas Propag. IEEE Trans. 2009, 57, 2949-2956. 
13. Beneduci, A. Evaluation of the Potential in vitro Antiproliferative Effects of Millimeter Waves at Some Therapeutic Frequencies on RPMI 7932 Human Skin Malignant Melanoma Cells. Cell Biochem. Biophys. 2009, 55, 25-32.

14. Zhadobov, M.; Sauleau, R.; Vie, V.; Himdi, M.; le Coq, L.; Thouroude, D. Interactions between 60-GHz millimeter waves and artificial biological membranes: Dependence on radiation parameters. Microw. Theory Tech. IEEE Trans. 2006, 54, 2534-2542.

15. Beneduci, A.; Filippelli, L.; Cosentino, K.; Calabrese, M.L.; Massa, R.; Chidichimo, G. Microwave induced shift of the main phase transition in phosphatidylcholine membranes. Bioelectrochemistry 2012, 84, 18-24.

16. Ramundo-Orlando, A.; Gallerano, G.P.; Stano, P.; Doria, A.; Giovenale, E.; Messina, G.; Cappelli, M.; D’Arienzo, M.; Spassovsky, I. Permeability changes induced by $130 \mathrm{GHz}$ pulsed radiation on cationic liposomes loaded with carbonic anhydrase. Bioelectromagnetics 2007, 28, 587-598.

17. Cosentino, K.; Beneduci, A.; Ramundo-Orlando, A.; Chidichimo, G. The influence of millimeter waves on the physical properties of large and giant unilamellar vesicles. J. Biol. Phys. 2013, 1-16.

18. Ramundo-Orlando, A.; Longo, G.; Cappelli, M.; Girasole, M.; Tarricone, L.; Beneduci, A.; Massa, R. The response of giant phospholipid vesicles to millimeter waves radiation. Biochim. Biophys. Acta 2009, 1788, 1497-1507.

19. Faure, C.; Bonakdar, L.; Dufourc, E.J. Determination of DMPC hydration in the $\mathrm{L}_{\alpha}$ and $\mathrm{L}_{\beta^{\prime}}$ phases by ${ }^{2} \mathrm{H}$ solid state NMR of $\mathrm{D}_{2} \mathrm{O}$. FEBS lett. 1997, 405, 263-266.

20. Pope, J.M.; Walker, L.; Cornell, B.A.; Francis, G.W. NMR study of synthetic lecithin bilayers in the vicinity of the gel-liquid-crystal transition. Biophys. J. 1981, 35, 509-520.

21. Strenk, L.M.; Westerman, P.W.; Doane, J.W. A model of orientational ordering in phosphatidylcholine bilayers based on conformational analysis of the glycerol backbone region. Biophys. J. 1985, 48, 765-773.

22. Massiot, D.; Fayon, F.; Capron, M.; King, I.; le Calvé, S.; Alonso, B.; Durand, J.-O.; Bujoli, B.; Gan, Z.; Hoatson, G. Modelling one- and two-dimensional solid-state NMR spectra. Magn. Reson. Chem. 2002, 40, 70-76.

23. Chidichimo, G.; Golemme, A.; Doane, J.W. Investigation of ribbon lyotropic structures by deuterium magnetic resonance. J. Chem. Phys. 1985, 82, 4369-4375.

24. Stockton, G.W.; Johnson, K.G.; Butler, K.W.; Tulloch, A.P.; Boulanger, Y.; Smith, I.C.P.; Davis, J.H.; Bloom, M. Deuterium NMR study of lipid organisation in Acholeplasma laidlawii membranes. Nature 1977, 296, 267-268.

25. Davis, J.H. The description of membrane lipid conformation, order and dynamics by ${ }^{2} \mathrm{H}-\mathrm{NMR}$. Biochim. Biophys. Acta (BBA) Rev. Biomembr. 1983, 737, 117-171.

26. Sparrman, T.; Westlund, P.-O. An NMR line shape and relaxation analysis of heavy water powder spectra of the $\mathrm{L}_{\alpha}, \mathrm{L}_{\beta^{\prime}}$ and $\mathrm{P}_{\beta^{\prime}}$ phases in the DPPC/water system. Phys. Chem. Chem. Phys. 2003, 5, 2114-2121.

27. Fröhlich, H. Biological Coherence and Response to External Stimuli; Springer-Verlag: Berlin, Germany, 1988. 
28. Reimers, J.R.; McKemmish, L.K.; McKenzie, R.H.; Mark, A.E.; Hush, N.S. Weak, strong, and coherent regimes of Fröhlich condensation and their applications to terahertz medicine and quantum consciousness. Proc. Nat. Acad. Sci. 2009, 106, 4219-4224.

29. Douliez, J.P.; Léonard, A.; Dufourc, E.J. Restatement of order parameters in biomembranes: Calculation of $\mathrm{C}-\mathrm{C}$ bond order parameters from $\mathrm{C}-\mathrm{D}$ quadrupolar splittings. Biophys. J. 1995, 68, 1727-1739.

(C) 2013 by the authors; licensee MDPI, Basel, Switzerland. This article is an open access article distributed under the terms and conditions of the Creative Commons Attribution license (http://creativecommons.org/licenses/by/3.0/). 